\title{
A NEW APPROACH TO THE MULTIPLIERS OF PEDERSEN'S IDEAL
}

\author{
N. CHRISTOPHER PHILLIPS
}

(Communicated by Paul S. Muhly)

\begin{abstract}
We obtain a simple description of the multiplier algebra $\Gamma\left(K_{A}\right)$ of Pedersen's ideal $K_{A}$ of a $C^{*}$-algebra $A$ as a pro- $C^{*}$-algebra (inverse limit of $C^{*}$-algebras). This description enables us to derive a number of the results obtained by Lazar and Taylor in their study of $\Gamma\left(K_{A}\right)$ directly from corresponding facts about pro- $C^{*}$-algebras, and to give simplified proofs of several other results.
\end{abstract}

Let $A$ be a $C^{*}$-algebra without identity. In [4], Pedersen introduced a minimal dense hereditary ideal $K_{A}$ of $A$, which has since been shown to be minimal among all dense ideals of $A$. (Compare [5, 5.6.1].) In [3], Lazar and Taylor made an extensive study of the multiplier algebra $\Gamma\left(K_{A}\right)$ of $A$. In this paper, we give the following explicit description of $\Gamma\left(K_{A}\right)$ in terms of the pro- $C^{*}$-algebras of [6]: the algebra $\Gamma\left(K_{A}\right)$ is isomorphic as a *-algebra to the inverse limit of the multiplier algebras $M(A / J)$ as $J$ runs through all closed ideals of $A$ such that the primitive ideal space $\operatorname{Prim}(A / J)$ is compact. If $J_{1} \subset J_{2}$ then the map $M\left(A / J_{1}\right) \rightarrow M\left(A / J_{2}\right)$ is obtained from the surjective map $A / J_{1} \rightarrow A / J_{2}$ as in Theorem 4.2 of [1]. (Of course, we do not claim that the map of the multiplier algebras is surjective in general.) Furthermore, if $A$ is $\sigma$-unital, that is, has a countable approximate identity, then the maps $\Gamma\left(K_{A}\right) \rightarrow M(A / J)$ are all surjective. This description enables us to obtain most of the functional calculus results in Chapter 5 of [3] as immediate corollaries of general facts about pro- $C^{*}$-algebras, and to give quick proofs of several other results from [3], such as the Dauns-Hofmann theorem for $\Gamma\left(K_{A}\right)$.

Our first step is to exhibit $\Gamma\left(K_{A}\right)$ as some inverse limit of $C^{*}$-algebras (Theorem $2)$. This result already has a large number of consequences. Next, we identify the algebras in the inverse system as certain multiplier algebras, obtaining Theorem 4. The description given in the previous paragraph is then obtained in Theorem 7 by replacing the multiplier algebras in Theorem 4 with smaller ones.

We will use Chapters 2 and 3 of [3] as a general reference for facts about the Pedersen ideal and multipliers of normed *-algebras. Following [3], for $a \in A$ we denote by $L_{a}$ the closed left ideal $\overline{A a}$ generated by $a$, and by $R_{a}$ the closed right ideal generated by $a$. (Note that $L_{a}$ and $R_{a}$ do in fact contain $a$.) We further denote by $M_{a}$ the $C^{*}$-algebra of all pairs $(S, T)$ consisting of linear maps $S: L_{a} \rightarrow L_{a}$ and $T: R_{a^{*}} \rightarrow R_{a^{*}}$ such that $y S(x)=T(y) x$ for $x \in L_{a}$ and $y \in R_{a^{*}}$. (Note that $S$

Received by the editors October 26, 1987.

1980 Mathematics Subject Classification (1985 Revision). Primary 46L05; Secondary 46K99, $46 \mathrm{M} 40$.

Key words and phrases. Pedersen's ideal, multiplier algebra, pro- $C^{*}$-algebra, inverse limit of $C^{*}$-algebras.

Research supported by an NSF postdoctoral fellowship. 
and $T$ are automatically bounded, and that $M_{a}$ is in fact a $C^{*}$-algebra, by Lemma 5.2 of $[3]$.) We further denote by $I_{a}$ the closed two-sided ideal $\overline{L_{a} R_{a^{*}}}$ generated by $a a^{*}$. The subscript + on a set will denote its subset of positive elements.

1. Lemma. Let $a, b \in A$ with $0 \leq a \leq b$. Then $L_{a} \subset L_{b}, R_{a} \subset R_{b}$, and $I_{a} \subset I_{b}$. Furthermore, the restriction map $(S, T) \mapsto\left(\left.S\right|_{L_{a}},\left.T\right|_{R_{a}}\right)$ defines a ${ }^{*}$-homomorphism from $M_{b}$ to $M_{a}$.

PROOF. For the first part, it suffices to show that $a \in L_{b} \cap R_{b}$. But $L_{b} \cap R_{b}$ is the closed hereditary subalgebra generated by $b[5,1.5 .2]$, hence contains $a$ by definition. The second part is now obvious. Q.E.D.

The following theorem has also been found by John Mack.

2. THEOREM. Let $A$ be a $C^{*}$-algebra. Then $\Gamma\left(K_{A}\right)$ is isomorphic as a*algebra to the pro- $C^{*}$-algebra $\lim _{a \in\left(K_{A}\right)_{+}} M_{a}$. Furthermore, the algebra of bounded elements $b\left(\Gamma\left(K_{A}\right)\right)$ of $\Gamma\left(K_{A}\right)[6$, Definition 1.9] is equal to $M(A)$.

Proof. The maps $\varphi_{a}: \Gamma\left(K_{A}\right) \rightarrow M_{a}$ are defined by restriction (as in [3, Definition 5.3], using the fact [3, Proposition 3.3] that for $a \in K_{A}$ we have $L_{a}, R_{a} \subset$ $K_{A}$, and the fact [3, Proposition 3.4] that, for $(S, T) \in \Gamma\left(K_{A}\right)$, we have $S\left(L_{a}\right) \subset L_{a}$ and $T\left(R_{a}\right) \subset R_{a}$. These maps obviously give a homomorphism $\varphi: \Gamma\left(K_{A}\right) \rightarrow$ $\lim _{a \in\left(K_{A}\right)} M_{a}$. To see that $\varphi$ is injective, note that if $\varphi(x)=0$ then $x a=0$ for every $a \in\left(K_{A}\right)_{+}$and hence every $a \in K_{A}$. This implies that $x=0$. To show that $\varphi$ is surjective, let $\left(\left(S_{a}, T_{a}\right)\right)_{a \in\left(K_{A}\right)_{+}}$be any coherent sequence of elements of the algebras $M_{a}$. Then define $S, T: K_{A} \rightarrow K_{A}$ by the requirements that $\left.S\right|_{L_{a}}=S_{a}$ and $\left.T\right|_{R_{a}}=T_{a}$. This makes sense because the maps $M_{b} \rightarrow M_{a}$ are defined by restriction, and because $\bigcup_{a \in\left(K_{A}\right)_{+}} L_{a}$ and $\bigcup_{a \in\left(K_{A}\right)_{+}} R_{a}$ are ${ }^{*}$-subalgebras of $K_{A}$ which contain $\left(K_{A}\right)_{+}$and are hence equal to $K_{A}$. The fact that $S$ and $T$ are linear and define a multiplier follows from the fact that, by the previous lemma, we have $L_{a}+L_{b} \subset L_{a+b}$. (It is unnecessary to verify the relations $S(x y)=S(x) y$ and $T(x y)=x T(y)$ - see [3, Lemma 2.10] and the remarks preceding it.) This shows that $\varphi$ is surjective.

It remains only to prove that $b\left(\Gamma\left(K_{A}\right)\right)=M(A)$. This is however clear from the definition of the norm in $M_{a}$ and from the argument above, because $M(A)$ is equal to the set of bounded multipliers of $K_{A}$. Q.E.D.

We point out that the pro- $C^{*}$ topology on $\Gamma\left(K_{A}\right)$, determined by the isomorphism of the theorem, is stronger than the $\kappa$-topology of [3], and is usually strictly stronger. We will say more about it in Theorems 4 and 7.

The results in Chapter 5 of [3] on the spectrum, on selfadjoint and positive elements, and on continuous functional calculus in $\Gamma\left(K_{A}\right)$ now follow immediately from the corresponding results for pro- $C^{*}$-algebras in $\S 1$ of [6]. Note, however, that the $\kappa$-topology must be replaced everywhere by the pro- $C^{*}$ topology. (This change makes the results stronger in most cases.) In fact, either directly from [6] or by applying the corresponding facts for $C^{*}$-algebras to coherent sequences in the inverse limit, we obtain 5.6 through 5.32 and 5.35 through 5.38 of [3], with the pro- $C^{*}$ topology, except for the parts of 5.6 and 5.8 involving the approximate identity, 5.10, 5.26, and the examples. (Note, however, that 5.10 and the parts of 5.6 and 5.8 involving the approximate identity will follow from Theorem 4 below.) We also obtain an affirmative answer to 5.33. 
To get more information, we need to identify the algebras $M_{a}$.

3. Proposition. Let $a \in A_{+}$. Then the map $\psi: M\left(I_{a}\right) \rightarrow M_{a}$, defined by $\psi(S, T)=\left(\left.S\right|_{L_{a}},\left.T\right|_{R_{a}}\right)$, is a ${ }^{*}$-isomorphism.

Proof. To show that $\psi$ is a ${ }^{*}$-homomorphism, we need only show that if $(S, T)$ is a multiplier of $I_{a}$, then $S\left(L_{a}\right) \subset L_{a}$ and $T\left(R_{a}\right) \subset R_{a}$. Since the proofs are the same, we do only the first of these. Let $x \in A$. Then $x a^{1 / 2} \in I_{a}$, so $S\left(x a^{1 / 2}\right)$ is defined. Furthermore, $a^{1 / 2} \in L_{a}$. (In fact, $a^{1 / 2} \in L_{a} \cap R_{a}$ because $L_{a} \cap R_{a}$ is a closed *-subalgebra of $A$ which contains $a$.) Therefore $S(x a)=S\left(\left(x a^{1 / 2}\right) a^{1 / 2}\right)=$ $S\left(x a^{1 / 2}\right) a^{1 / 2} \in L_{a}$. By continuity, we obtain $S\left(L_{a}\right) \subset L_{a}$.

Next observe that $\psi$ is injective. Indeed, if $\psi(S, T)=0$ then $\left.S\right|_{L_{a}}=0$. Therefore the property $S(x y)=S(x) y$, for $x \in L_{a}$ and $y \in R_{a}$, implies that $\left.S\right|_{L_{a} R_{a}}=0$. Since $L_{a} R_{a}$ is dense in $I_{a}$, we obtain $S=0$. It follows that $T=0$.

We now show that $\psi\left(I_{a}\right)$ is an ideal in $M_{a}$. By linearity and continuity, it is enough to show that if $z=x a^{2} y \in L_{a} R_{a}$, then $\psi(z) M_{a}, M_{a} \psi(z) \subset \psi\left(I_{a}\right)$. Again, we do only one of these. Note that $\psi(z)=\left(l_{z}, r_{z}\right)$, where $l_{z}(b)=z b$ and $r_{z}(c)=c z$. Then for $(S, T) \in M_{a}$ and $b \in L_{a}$, we have

$$
\left(l_{z} \circ S\right)(b)=x a^{2} y S(b)=x a T(a y) b=l_{x a T(a y)}(b) .
$$

Furthermore, for $c \in R_{a}$ and $d \in L_{a}$, we have

$$
\left(T \circ r_{z}\right)(c) d=T\left(c x a^{2} y\right) d=c x a^{2} y S(d)=c x a T(a y) d .
$$

Putting $d=\left(\left(T \circ r_{z}\right)(c)-c x a T(a y)\right)^{*}$, we obtain $d d^{*}=0$, whence

$$
\left(T \circ r_{z}\right)(c)=c x a T(a y)=r_{x a T(a y)}(c) \text {. }
$$

Thus, $\psi(z)(S, T)=\psi(x a T(a y)) \in \psi\left(I_{a}\right)$. It follows that $\psi\left(I_{a}\right)$ is an ideal in $M_{a}$.

Next, we note that $\psi\left(I_{a}\right)$ is essential. If for some $(S, T) \in M_{a}$, we have $\psi\left(I_{a}\right) \cdot(S, T)=0$, then in particular, using the calculations above, we obtain $r_{x a T(a y)}=0$ for all $x, y \in A$. In particular, $a^{3} T(a y)=r_{a^{2} T(a y)}(a)=0$ for all $y \in A$. Functional calculus gives $a^{1 / n} T(a y)=0$ for all $n$. Since $\left(a^{1 / n}\right)$ is a left approximate identity for $R_{a}$ (see [3, Lemma 3.2]), we obtain $T(a y)=0$, so, by continuity, $T=0$. Since $\|T\|=\|S\|$, we obtain $S=0$ also.

The standard properties of multiplier algebras now yield an injective map $\rho: M_{a} \rightarrow M\left(I_{a}\right)$ defined by $\rho(x)=\left(l_{x}, r_{x}\right)$, where $l_{x}(c)=\psi^{-1}(x \psi(c))$ and $r_{x}(c)=$ $\psi^{-1}(\psi(c) x)$. Obviously $\psi \circ \rho$ is the identity on $M_{a}$. Since both $\psi$ and $\rho$ are injective, it follows that $\psi$ and $\rho$ are in fact isomorphisms. Q.E.D.

4. THEOREM. Let $A$ be a $C^{*}$-algebra. Then, for any approximate identity $\left(e_{\lambda}\right)$ for $A$ contained in $K_{A}$, we have

$$
\Gamma\left(K_{A}\right) \cong \underbrace{\lim }_{a \in\left(K_{A}\right)+} M\left(I_{a}\right) \cong \lim _{\lambda}^{\lim } M\left(I_{e_{\lambda}}\right) .
$$

In particular, if $A$ is $\sigma$-unital, then $\Gamma\left(K_{A}\right)$ is a $\sigma-C^{*}$-algebra. The inverse limit of the strict topologies on the algebras $M\left(I_{a}\right)$ is stronger than the $\kappa$-topology, but agrees with the $\kappa$-topology on subsets of $\Gamma\left(K_{A}\right)$ which are bounded (in the topological vector space sense) for the pro- $C^{*}$ topology.

PROOF. That $\Gamma\left(K_{A}\right) \cong \lim _{a \in\left(K_{A}\right)_{+}} M\left(I_{a}\right)$ is immediate from the previous proposition and Theorem 2. Furthermore, we can obviously replace the directed 
set $\left(K_{A}\right)_{+}$by the set $\left\{I_{a}: a \in\left(K_{A}\right)_{+}\right\}$, ordered by inclusion. The isomorphism $\Gamma\left(K_{A}\right) \cong \lim _{\lambda} M\left(I_{e_{\lambda}}\right)$ will then follow if we can show that for every $a \in\left(K_{A}\right)_{+}$ there is $\lambda$ such that $I_{a} \subset I_{e_{\lambda}}$. Now consider the set $\bigcup_{\lambda} I_{e_{\lambda}}$. It is an ideal in $A$ by Lemma 1 , and it is obviously dense, so it contains $K_{A}$. Thus $a \in I_{e_{\lambda}}$ for some $\lambda$, whence $I_{a} \subset I_{e_{\lambda}}$ as desired.

It is now immediate that $\Gamma\left(K_{A}\right)$ is a $\sigma$ - $C^{*}$-algebra if $A$ has a countable approximate identity.

For the statement about topologies, let $\left(x_{\alpha}\right)$ be a net in $\lim _{\longleftarrow} M\left(I_{a}\right)$. If $x_{\alpha} \rightarrow x$ in the inverse limit of the strict topologies, then, using $a \in I_{a}$ for $a \in\left(K_{A}\right)_{+}$, we obtain in particular $x_{\alpha} a \rightarrow x a$ and $a x_{\alpha} \rightarrow a x$ in norm. Since $K_{A}$ is spanned by its positive elements, we see that $x_{\alpha} \rightarrow x$ in the $\kappa$-topology. Now assume that $\left(x_{\alpha}\right)$ is bounded, and let $x_{\alpha} \rightarrow x$ in the $\kappa$-topology. Let $a \in\left(K_{A}\right)_{+}$and let $\pi: \Gamma\left(K_{A}\right) \rightarrow M\left(I_{a}\right)$ be the canonical map. Then $\left(\pi\left(x_{\alpha}\right)\right)$ is norm-bounded in $M\left(I_{a}\right)$ and, for $b \in K_{A} \cap I_{a}$, we have $\pi\left(x_{\alpha}\right) b \rightarrow \pi(x) b$ and $b \pi\left(x_{\alpha}\right) \rightarrow b \pi(x)$. Since $K_{A} \cap I_{a}$ is norm-dense in $I_{a}$ (it contains $L_{a} R_{a}$ ), we see that $\pi\left(x_{\alpha}\right) \rightarrow \pi(x)$ strictly, as desired. Q.E.D.

The inverse systems used in Theorem 4 have the disadvantage that the maps from $\Gamma\left(K_{A}\right)$ to the algebras appearing in the inverse system are usually not surjective. We will now show how to correct that difficulty, at least for $\sigma$-unital algebras.

5. LeMma. (1) For $a \in\left(K_{A}\right)_{+}$, the closure of $\operatorname{Prim}\left(I_{a}\right)$ in $\operatorname{Prim}(A)$ is compact.

(2) If $C \subset \operatorname{Prim}(A)$ is any compact set, then there exists $a \in\left(K_{A}\right)+$ such that $C \subset \operatorname{Prim}\left(I_{a}\right)$.

ProOF. Part (1) follows immediately from Lemma 5.39 of [3]. (Note that the proof of this lemma uses only the definition of $K_{A}$ and [2,3.3.7], not the other results in Chapter 5 of [3].) To prove part (2), for each $P \in C$ choose $a_{P} \in\left(K_{A}\right)_{+}$such that $\left\|a_{P}+P\right\|>1$. (This can be done since $\left(K_{A}\right)_{+}$is dense in $A_{+}$.) Cover $C$ with finitely many of the sets $\left\{Q \in C:\left\|a_{P}+Q\right\|>1\right\}$. (These sets are open by [2, 3.3.2].) Let $P_{1}, \ldots, P_{n}$ be the corresponding primitive ideals, and set $a=a_{P_{1}}+\cdots+a_{P_{n}}$. Then $\|a+Q\|>1$ for every $Q \in C$, so that $C \subset \operatorname{Prim}\left(I_{a}\right)$. Q.E.D.

The next lemma appears in [3], but we can give a simpler proof here.

6. LEMMA [3, COROLLARY 10.8]. Let $A$ be a $C^{*}$-algebra whose primitive ideal space is compact. Then $\Gamma\left(K_{A}\right)=M(A)$, that is, every multiplier of $K_{A}$ is continuous.

ProOF. By part (2) of the previous lemma, we can choose $a \in\left(K_{A}\right)_{+}$such that $I_{a}=A$. Theorem 4 now implies that $\Gamma\left(K_{A}\right)=M\left(I_{a}\right)=M(A)$. Q.E.D.

Note that the element $a$ used in this proof is the one constructed in the proof of Corollary 10.8 of [3], What has really happened is that, using Proposition 3, we are able to obtain continuity of a multiplier of $K_{A}$ directly from Lemma 5.2 of [3], without using the complicated Theorems 10.1 and 10.7 of [3].

The next theorem is our most revealing description of $\Gamma\left(K_{A}\right)$. We denote by $I(U)$ the closed ideal in $A$ corresponding to an open subset $U$ of $\operatorname{Prim}(A)$. We also denote the multiplier algebra of a pro- $C^{*}$-algebra $B$ by $M(B)$; it is defined in $\S 2$ of [6], as is the strict topology on it. 
7. THEOREM. Let $A$ be a $C^{*}$-algebra with primitive ideal space $X$. Then

$$
\Gamma\left(K_{A}\right) \cong \lim _{C} M(A / I(X-C)) \cong M\left({\underset{\lim }{C}}_{C} A / I(X-C)\right),
$$

where the inverse limits run over the set of compact closed subsets $C$ of $X$, ordered by inclusion. If $A$ is $\sigma$-unital, then the maps

$$
\Gamma\left(K_{A}\right) \rightarrow M(A / I(X-C))
$$

are all surjective. The strict topology on $M(\lim A / I(X-C))$ is stronger than the $\kappa$ topology, but agrees with the $\kappa$-topology on subsets which are bounded for the pro- $C^{*}$ topology.

Proof. We first show that $\Gamma\left(K_{A}\right) \cong \lim _{C} M(A / I(X-C))$. For $a \in K_{A}$, let $C_{a}=\overline{\operatorname{Prim}\left(I_{a}\right)}$, which is a compact subset of Prim $(A)$ by Lemma $5(1)$, and let $J_{a}=I\left(X-C_{a}\right)$. In view of Lemma 5(2), the sets $C_{a}$ are cofinal in the collection of all compact closed subsets of $X$. By Theorem 4, it is therefore enough to show that the map $\Gamma\left(K_{A}\right) \rightarrow M\left(I_{a}\right)$ factors canonically through $M\left(A / J_{a}\right)$. Now Propositions 2.9 and 2.13 of [3] yield a homomorphism from $\Gamma\left(K_{A}\right)$ to $\Gamma\left(K_{A / J_{a}}\right)$, and by Lemma 6 , we have $\Gamma\left(K_{A / J_{a}}\right)=M\left(A / J_{a}\right)$. We furthermore have a map $M\left(A / J_{a}\right) \rightarrow M\left(I_{a}\right)$ obtained from the fact that $I_{a}$ is an ideal in $A / J_{a}$. (The quotient map $A \rightarrow A / J_{a}$ sends $I_{a}$ to $\left(I_{a}+J_{a}\right) / J_{a} \cong I_{a} /\left(I_{a} \cap J_{a}\right)$, but $I_{a} \cap J_{a}=\{0\}$.) It is easily shown that the composite of these two maps is in fact the map from $\Gamma\left(K_{A}\right)$ to $M\left(I_{a}\right)$. So the isomorphism $\Gamma\left(K_{A}\right) \cong \lim _{C} M(A / I(X-C))$ is proved.

To show that $\Gamma\left(K_{A}\right) \rightarrow M(A / I(X-C))$ is surjective when $A$ is $\sigma$-unital, we observe that already $M(A) \rightarrow M(A / I(X-C))$ is surjective, by [6, Theorem 5.11].

The isomorphism $M\left(\lim _{C} A / I(X-C)\right) \cong \varliminf_{C} M(A / I(X-C))$ follows from Theorem 3.14(1) of [6]. (Note that the inverse limit ${\underset{\lim }{C}}_{C} A / I(X-C)$ certainly contains $A$, so that the maps from the inverse limit to each $A / I(X-C)$ are in fact all surjective.)

It remains only to prove the statement about the topologies. It is sufficient to show that $K_{A}$ is dense in $\lim A / I(X-C)$ for the pro- $C^{*}$ topology. This in turn will follow from the norm density of the image of $K_{A}$, in each $A / I(X-C)$. But according to [3, Proposition 2.9], the image of $K_{A}$ in $A / I(X-C)$ is the dense ideal $K_{A / I(X-C)}$. Q.E.D.

The last paragraph of the proof above shows that $K_{A}$ is also a dense ideal in the pro- $C^{*}$-algebra $\lim _{C} A / I(\operatorname{Prim}(A)-C)$. Since this algebra might be unital, for example if $A=C_{0} \overleftarrow{(X)}$, the algebra of continuous functions vanishing at infinity on the locally compact Hausdorff space $X$, we have another illustration of the fact that the group of invertible elements of a unital pro- $C^{*}$-algebra need not be open.

As in [3], we regard a nonunital $C^{*}$-algebra $A$ as a noncommutative analog of $C_{0}(X)$. Then $M(A)$ becomes the noncommutative analog of the algebra $C_{b}(X)$ of bounded continuous functions, $K_{A}$ becomes the analog of the algebra $C_{c}(X)$ of compactly supported continuous functions, and $\Gamma\left(K_{A}\right)$ becomes the analog of the algebra $C(X)$ of all continuous functions. The pro- $C^{*}$-algebra

$$
\varliminf_{C}^{\lim } A / I(\operatorname{Prim}(A)-C)
$$


can then be regarded as the continuous functions which vanish at infinity in the "nonconmmutative direction" but are unrestricted in the "commutative direction". Similar interpretations can be given to other algebras one might construct, such as $\bigcup_{a \in\left(K_{A}\right)_{+}} I_{a}$ and $\lim _{C} K_{A / I(\operatorname{Prim}(A)-C)}$. With this interpretation, Lemma 6 and Theorem 7 assert that every continuous function is automatically bounded in the "noncommutative direction".

We now give a simplified proof of the Dauns-Hofmann theorem for $K_{A}$, Theorem 5.42 of $[3]$. As usual, we use the pro- $C^{*}$ topology instead of the $\kappa$-topology.

8. THEOREM [3, THEOREM 5.42]. Let $A$ be a $C^{*}$-algebra. Then the center $Z\left(\Gamma\left(K_{A}\right)\right)$ of $\Gamma\left(K_{A}\right)$ is isomorphic, as a pro- $C^{*}$-algebra, to $C(\operatorname{Prim}(A))$.

Proof. Let $X=\operatorname{Prim}(A)$. First note that $C(X)$ is a pro- $C^{*}$-algebra, because $X$ is locally compact and hence compactly generated (even though it need not be Hausdorff). By the usual Dauns-Hofmann theorem, it is enough to show that

$$
Z\left(\Gamma\left(K_{A}\right)\right)={\underset{\lim }{C}}_{C} Z(M(A / I(X-C)))
$$

where as usual $C$ runs through all compact closed subsets of $X$. Consider the map $M\left(A / I\left(X-C_{1}\right)\right) \rightarrow M\left(A / I\left(X-C_{2}\right)\right)$, where $C_{2} \subset C_{1}$. Its image contains $A / I\left(X-C_{2}\right)$, which is strictly dense in $M\left(A / I\left(X-C_{2}\right)\right)$. Since multiplication is separately strictly continuous, we conclude that the image of $Z\left(M\left(A / I\left(X-C_{1}\right)\right)\right)$ is contained in $Z\left(M\left(A / I\left(X-C_{2}\right)\right)\right)$. Therefore the inverse limit in (*) makes sense. The right-hand side of $(*)$ then obviously defines a subset of $Z\left(\Gamma\left(K_{A}\right)\right)$. To prove the reverse inclusion, it is obviously enough to show that the image of $Z\left(\Gamma\left(K_{A}\right)\right)$ in each $M(A / I(X-C))$ is contained in the center. This follows from the same argument used above to prove that the image of $Z\left(M\left(A / I\left(X-C_{1}\right)\right)\right)$ is contained in $Z\left(M\left(A / I\left(X-C_{2}\right)\right)\right)$. Q.E.D.

We conclude by taking another look at the question of when $\Gamma\left(K_{A}\right)=M(A)$. Lazar and Taylor asked whether or not it is true that $\Gamma\left(K_{A}\right)=M(A)$ as an algebra if and only if $\operatorname{Prim}(A)$ is pseudocompact, that is, every continuous complexvalued function on $\operatorname{Prim}(A)$ is bounded. Theorem 10.14 of [3] suggests that the answer should be yes. John Mack [7] has shown that the correct condition for $\Gamma\left(K_{A}\right)=M(A)$ is that $\operatorname{Prim}(A)$ be weakly compact. However, $\Gamma\left(K_{A}\right)$ is topologically isomorphic to $M(A)$ if and only if $\operatorname{Prim}(A)$ is actually compact.

9. Proposition. The natural map $M(A) \rightarrow \Gamma\left(K_{A}\right)$ is an isomorphism of pro- $C^{*}$-algebras if and only if $\operatorname{Prim}(A)$ is compact.

PROOF. The "if" part follows from Theorem 7. For the "only if" part, it suffices to produce, for every $C^{*}$-algebra $A$ whose primitive ideal space is not compact, a net in $M(A)$ which does not converge in $M(A)$ but which converges to zero in $\Gamma\left(K_{A}\right)$. The index set will be the set of all closed compact subsets $C$ of $\operatorname{Prim}(A)$, ordered by inclusion, and for such a set $C$ we let $a_{C} \in I(\operatorname{Prim}(A)-C)$ be any element of norm 1 . The net $\left(a_{C}\right)$ satisfies the required properties. Q.E.D.

\section{REFERENCES}

1. C. A. Akemann, G. K. Pedersen, and J. Tomiyama, Multipliers of $C^{*}$-algebras, J. Funct. Anal. 13 (1973), 277-301.

2. J. Dixmier, $C^{*}$-algebras, North-Holland, Amsterdam, New York, Oxford, 1977. 
3. A. J. Lazar and D. C. Taylor, Multipliers of Pedersen's ideal, Mem. Amer. Math. Soc. No. 169 (1976).

4. G. K. Pedersen, Measure theory for $C^{*}$-algebras, Math. Scand. 19 (1966), 131-145.

5. __,$C^{*}$-algebras and their automorphism groups, Academic Press, London, New York, San Francisco, 1979.

6. N. C. Phillips, Inverse limits of $C^{*}$-algebras, J. Operator Theory 19 (1988), 159-195.

7. J. Mack, The spectrum of a PCS-algebra, preprint.

Department of Mathematics, University of California, los Angeles, CaliFORNIA 90024

Current address: Department of Mathematics, University of Georgia, Athens Georgia 30602 